\title{
An Efficient Real Time Query System for Public Transportation Service using Zigbee and RFID
}

\author{
S.P. Manikandan and P. Balakrishnan
}

\begin{abstract}
In recent decades, the number of vehicles in India has increased enormously, which results in rigorous traffic congestion and pollution in urban areas, especially during peak hours. This paper describes an implementation of Real Time Query System (RTQS) for public transportation service using ZigBee and Radio Frequency Identification (RFID) as a communication medium. The System is a Demand Responsive Transit (DRT) but it is more competent and suitable as it entertains passenger's demands and gives data such as bus locations, bus number, and number of persons inside the bus in real time. The real time synchronization of this system makes this system more informative and distinctive when compared with other DRTs. Moreover, this system is more flexible and efficient when compared with the conventional systems. This paper discusses the usage of wireless technologies in real time query system and how to make it more reliable using wireless technology ZigBee and RFID.
\end{abstract}

Keywords--- RTQS, ZigBee, RFID, Microcontroller, IR, Voice Processor

\section{INTRODUCTION}

$\mathrm{I}$ $\mathrm{N}$ recent years there have been extreme changes in the field of transportation in India due to various aspects such as growing economy, rising levels of vehicle ownership and the public's expectation for advanced infrastructure and services [1]. At present, the bus travelers do not have enough information to choose their buses to their destination. The other main causes are the requirement to address uncoordinated planning of land use and transport, constraints in transport infrastructure expansion, an incompetent and insufficient public transport system [2]. In order to deal with these alterations and growing demands, a number of infrastructure expansion projects are being designed and implemented.

Wireless technologies play a vital role especially in the fields of medical, military and navy [3]. In earlier years, Walkie Talkie was used for transferring the data between the destinations. This wireless technique has been developed for industrial automation and it has shown its attention towards the transportation [4]. "Real Time Query System" for passengers in public transport system by using a wireless technology called "ZigBee" which is more efficient, reliable

S.P. Manikandan, Department of Electronics and Communication Engineering, K.S.R. College of Engineering, Tiruchengode.

P. Balakrishnan, Department of Electronics and Communication Engineering, K.S.R. College of Engineering, Tiruchengode. and less expensive [5]. This investigation is not the only method to develop this type of a system but it can absolutely be one of the better alternatives when compared with the conventional systems and can be employed in the areas where there are no 3G, WiMax or other long range wireless technologies available. This investigation clearly describes the efficiency and the competence of ZigBee. Till now, ZigBee is being used as in-house or in-vehicle technology but this investigation provides an idea of using ZigBee as communication tool for inter-vehicle and vehicle to infrastructure. ZigBee technology is used to communicate between Bus and the Bus Stop and ti will minimize the total cost of the system as ZigBee devices are far cheaper than Wi$\mathrm{Fi}$, 3G and WiMax devices [6]. As ZigBee is low power as compared to other wireless technologies like Wi-Fi, this system can be employed in mountainous regions where power is a major factor to be considered [7].

Generally GPS is employed to track the location of the vehicle and the information is updated in internet for accessing [8]. As the information is stored in internet, it can be accessed by everyone, but there is no use in providing the information to everyone as the people who are using the specific location can only be profited. In this system, the same data such as bus route, bus location, bus number, passenger count in bus is obtained without using GPS and internet in a cost effective manner.

For a long time in urban areas there are several issues faced by the people due to traffic conditions. However, recently, the urban transportation system has been developed because of the arrival of the wireless technology [9]. The public transportation system will dispatch and command the people about the public traffic vehicles automatically along with the time of vehicles via the electronic bus stop boards on the basis of optimization network layout, lines, public transportation mode configuration, station arrangement, departure interval determination and pricing, etc, thus reducing the travel time of passengers [10, 11]. The implementation of the real time query system and the construction of intelligent public transportation system must be under the assistance of innovative ideas and the support of sophisticated techniques [12,13]. The Public transportation system has a big place in the entire urban areas. The intelligent public transportation based on ZigBee and RFID will become an active area of research in industrial applications. Now, certain public transport companies have begun to build intelligent public transportation systems with real time query system $[14,15]$.

This real time query system offers the important bus information i.e. it contains the bus route number, no of 
passengers inside the bus, previous stop notification. In order to offer the essential bus information to all commuters, this paper proposes a real time query system in which all necessary information of buses will be gathered, processed, and presented to passengers when requested.

\section{SYSTEM ARCHITECTURE}

The RTQS has two modules namely bus module and bus stop module. Both modules use ZigBee and RFID as a communication medium.

\subsection{Bus Stop Module}

One of the essential modules in RTQS is bus stop module. Bus stop module utilize ATMEL microcontroller, which controls the whole block in the bus stop module. ZigBee Transceiver is employed as a bridge between bus module and bus stop module. ZigBee, which is present in this module transmits query from the bus stop module and receive response from the bus module. Keypad acts as interface between passenger and RTQS. Passengers can enter their query through the keypad present in the bus stop module. Serial interface connects ZigBee transceiver with microcontroller.

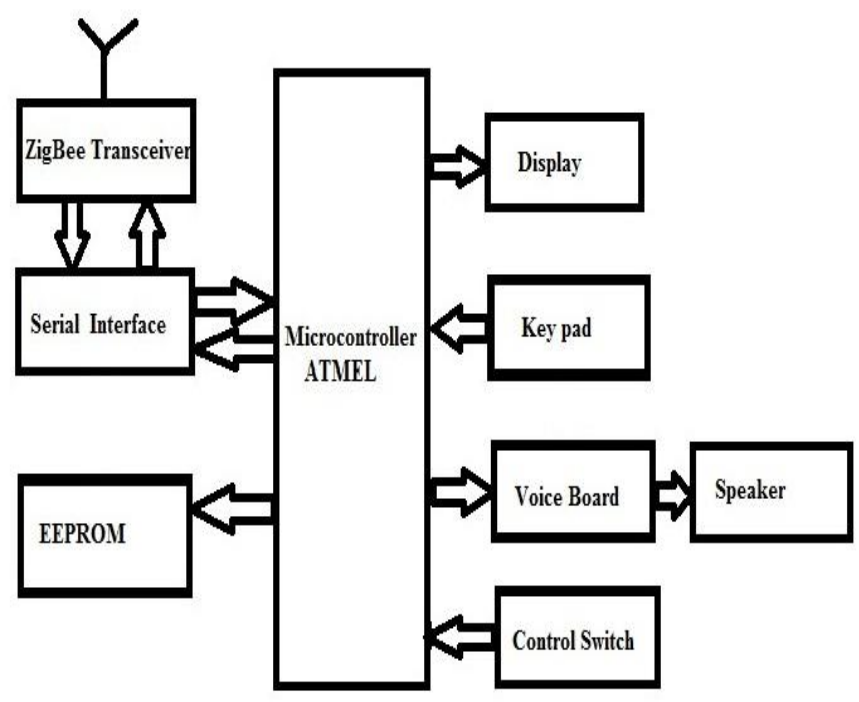

Figure 1: Block Diagram of Bus Stop Module

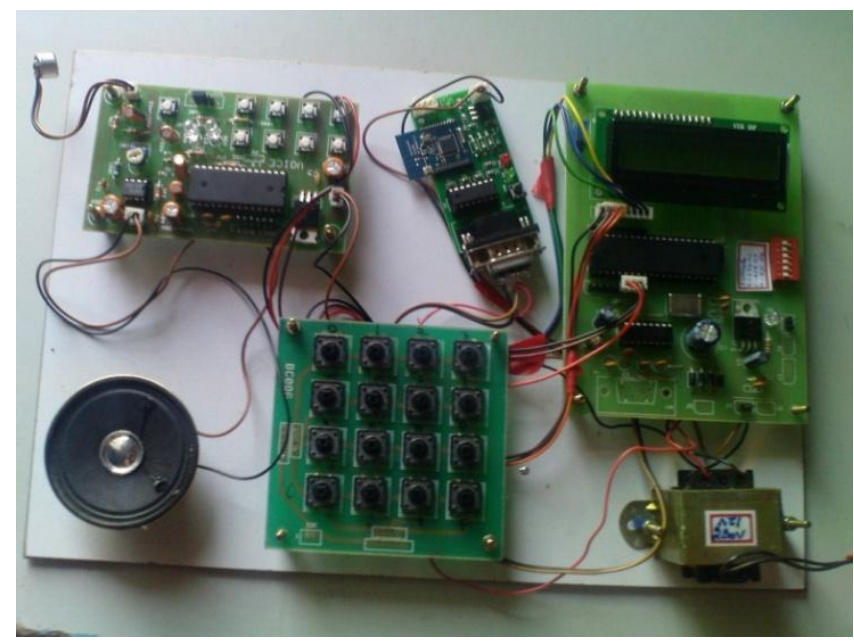

Figure 2: Bus Stop Module

The bus stop module also has EEPROM device. This device is used in this module, to store the database related to the bus number and destination. Voice board controls the speaker. The response transmitted from the bus module get received by the bus stop module and announced with the help of voice board and speaker. LCD display is used to display the response, which is received from the bus module. Control switch is used to choose the operating mode of bus stop module.

\subsection{Bus Module}

The second module present in the RTQS is bus module. Bus module also use ATMEL microcontroller, which control the whole block in the bus module. ZigBee Transceiver is used as bridge between bus module and bus stop module. ZigBee, which is present in this module receive query from the bus stop module and transmit response to the bus stop module.ZigBee is connected to microcontroller through serial interface.

IR sensor is placed in the bus module to count the number of persons inside the bus. These sensors are placed in the steps of the bus. IR sensors connect to the microcontroller through signal interface. Display unit present in the bus module is used to display the next bus stop. This entire block is placed inside the bus.

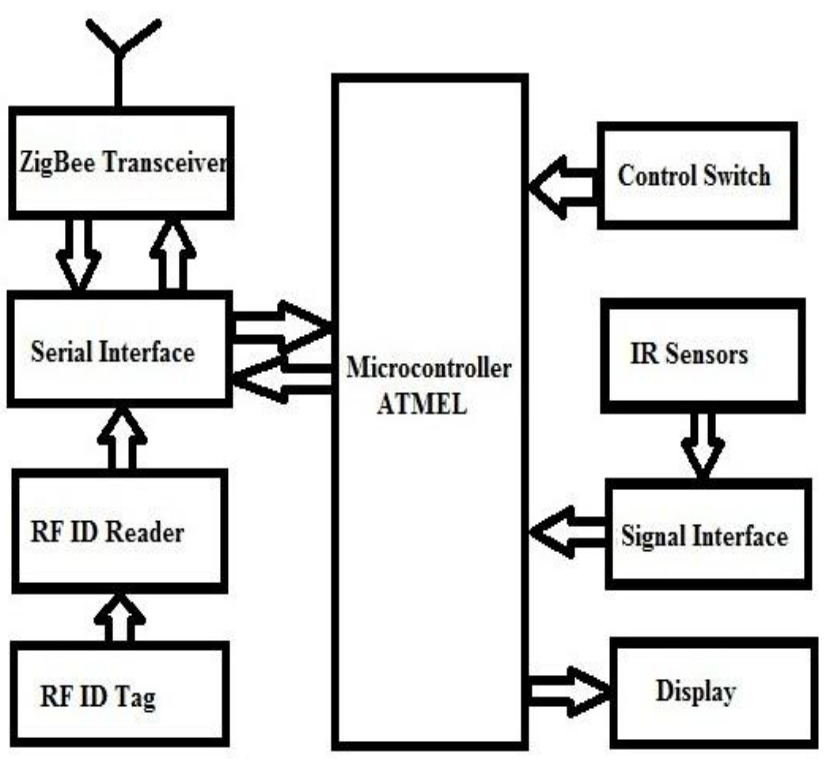

Figure 3: Block diagram of Bus module

Each bus stop has unique bus stop ID.This bus stop ID is stored in RFID tag. Each bus stop has its own RFID tag to hold the bus stop ID. Bus module has the RF ID reader to read the bus stop ID. This previously read bus stop ID will be transmitted to the requested bus stop as a response. Thus the proposed system consists of simplest and low cost device which reduce the overall system cost. 


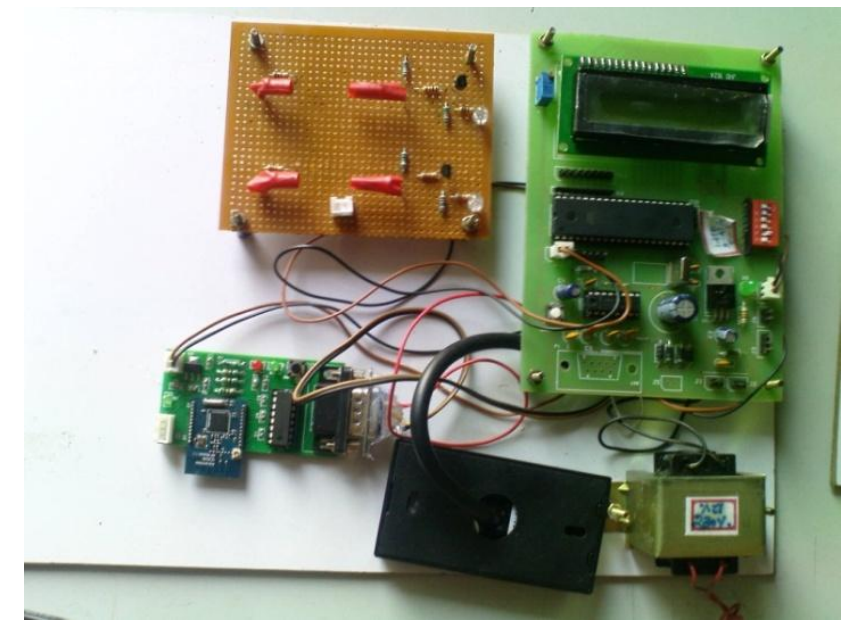

Figure 4: Bus Module

\section{MODES OF OPERATION}

The RTQS can be operated in two modes of operation

1. User query mode

2. Automatic Query mode

User query mode can be further divided in to two

1. User query based on bus route number

2. User query based on destination

\subsection{User Query Based on Bus Route Number}

In this mode of operation both bus stop and bus modules are involved. The user enters the desired bus route number (for instance 17D) with the help of key pad present in the bus stop module. They can also choose the type of bus in which he wishes to travel. Available types of buses are A/C or Deluxe or Regular. By default 'Regular' bus will be selected for the bus route number entered. A CANCEL button is included to change the selection and an ENTER key is used to confirm the entry. Then the query is transmitted through the ZigBee transceiver. The response is received from the nearest buses and the data is displayed on the LCD display on the bus stop module.

The bus route number is stored in each bus module that is fixed inside the bus. This bus module has a ZigBee transceiver to receive the query that was transmitted from the bus stop module. On receiving the query, the bus module compares the bus number specified in the query with its own bus number stored inside. If there is a match, the bus module transmits a response to the query. The response consists of two parts one is the bus stop id that is stored in the bus (i.e. the id of the bus stop which the bus has crossed previously) and another one is the crowd information (number of persons inside the bus).

\subsection{User Query Based on Destination}

Those passengers who do not know the required bus route number for their destination, they can get the information of bus route number just by typing the destination code in the RTQS. The user enters the desired destination code (for instance 5 for Ashok Pillar or 1 for T Nagar). A CANCEL button is included to change the selection and an ENTER key is used to confirm the entry. The bus stop module then searches the database that is stored in the EEPROM and filters and displays the buses that operate between the current bus stop and the required destination on the LCD display.

\subsection{Automatic Query Mode}

The bus stop module continuously sends queries. The buses having the bus stop id number that corresponds to the previous bus stop responds to the query by sending the bus route number to the querying bus stop module. This list is read out by using the voice board and the loud speaker.

\section{DIRECTION IDENTIFICATION}

Each bus stop is given a RF Tag specifying the corresponding direction code and bus stop code (For instance, $01,02 \ldots 0 \mathrm{~N}$ for the forward direction and $11,12 \ldots 1 \mathrm{~N}$ for the opposite or reverse direction). In the RF Tag, the first bit represents the direction: 0-forward direction and 1-opposite or reverse direction. And the remaining bits represent the actual bus stop number. The buses have their route numbers (for instance 17D, M70) programmed along with the category (A/C, Deluxe or regular) to which they belong to, inside the bus module. The buses as they cross a bus stop, the RF Tag of the bus stop is stored inside the bus module in the internal memory. As the bus keeps moving it will hold the RF ID of the last bus stop it had crossed. When it is moving in forward direction, it may have the tags $01,02,03 \ldots \ldots, 0 \mathrm{~N}$ till the last one it had crossed and $1 \mathrm{~N} \ldots \ldots ., 13,12,11$ for the reverse direction as it moves. So if a query is sent from the bus stop with RF Tag 03, for a particular bus, the bus routes traversing in both the direction may respond to the query and send its information to the bus stop module. In the stop module, the responses from the buses that have their previous bus stop ID starting with 0 alone are filtered out and the information is given to the user.

\section{CROWD INFORMATION}

In the bus one side of steps are used for entry and the other side of steps are used for exit. A sensor placed at both the sides is used to count the number of persons entered and the persons who exited and calculate the number of persons at that instant inside the bus. This information is stored inside the bus module. The response given by the bus module for crowd information will be based on the following scale

$<60$ persons - not crowded

$>60$ and $<75$ persons - almost crowded

$>75$ persons - crowded

\section{FlOW DIAGRAM}

Bus and bus stop module operations are represented as a separate flow diagram.

Steps in Bus stop modules are

3. Transmit query to the bus modules. The query consist of Bus number

4. Receive the response from the bus module whose bus number match with query's bus number

5. Display the response in the LCD and announce the same via voice board 


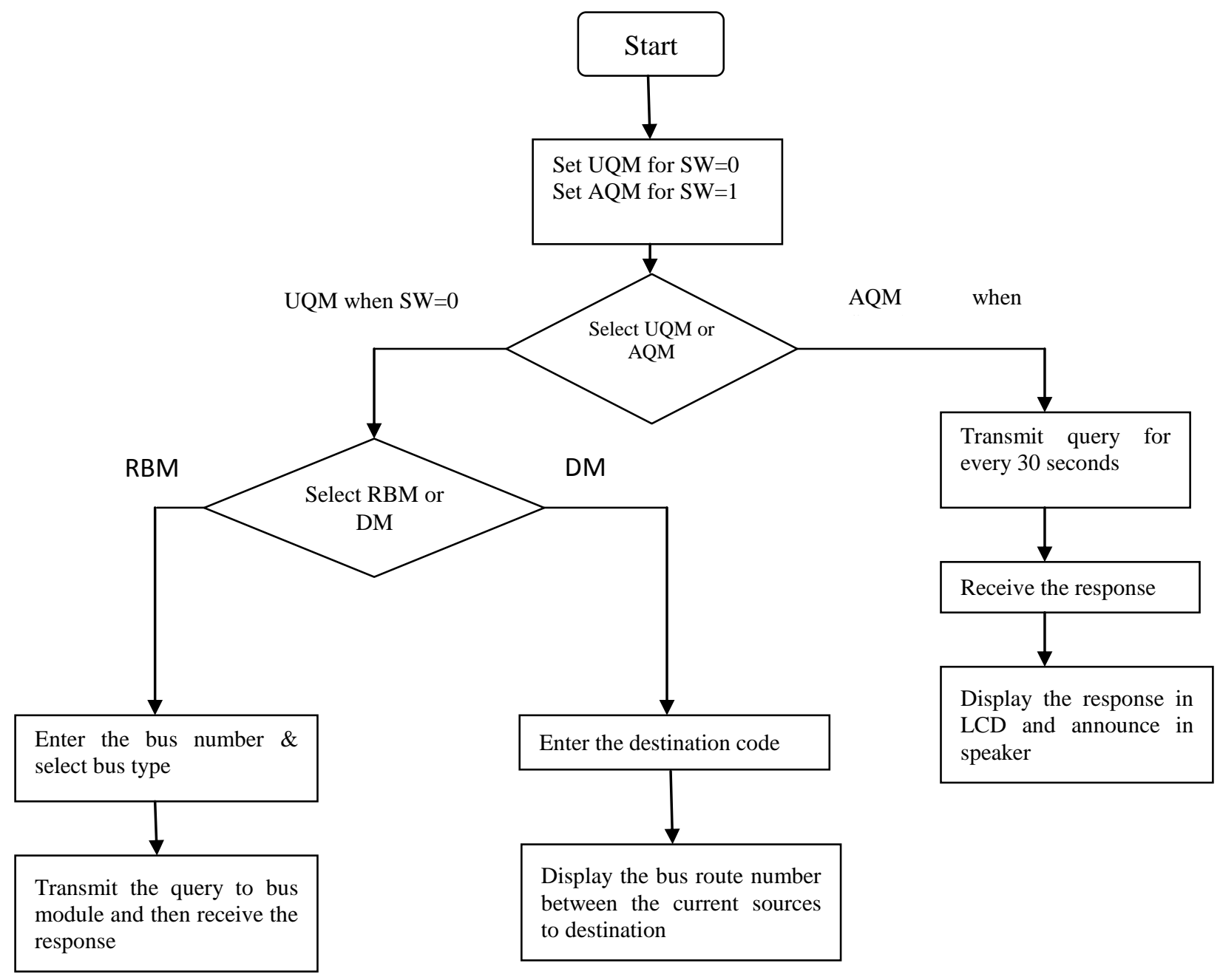

Figure 5: Flow chart of Bus Stop Module

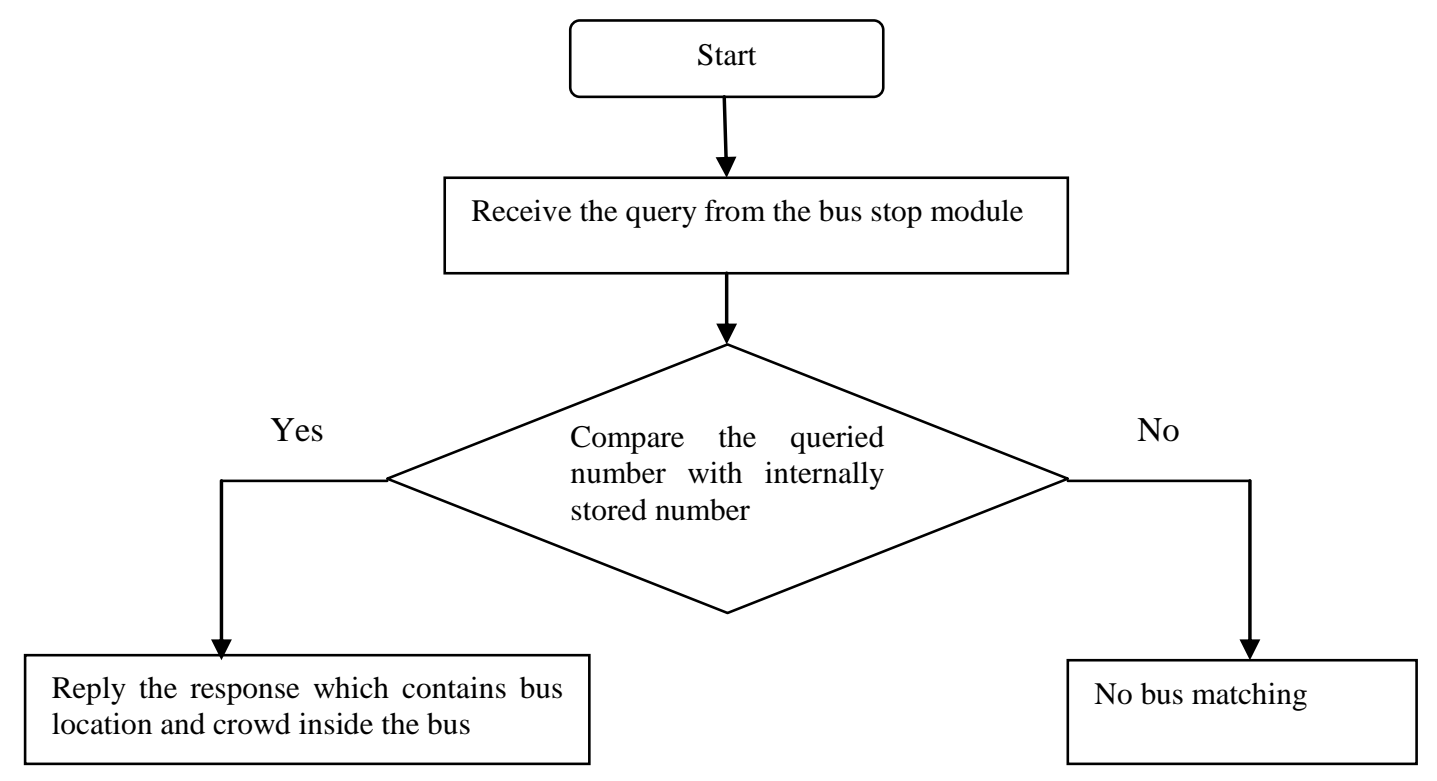

Figure 6: Flow Chart of Bus Module 
Steps in bus modules are

a. Receive query which was transmitted from the bus stop module

b. Check whether the requested bus number matches the bus number stored in the corresponding bus module

c. If it matches then the bus module respond the bus stop module by sending the response to the requested bus stop module. The response consist of previously crossed bus stop id and number of passengers inside the bus

\section{OVERVIEW OF RTQS}

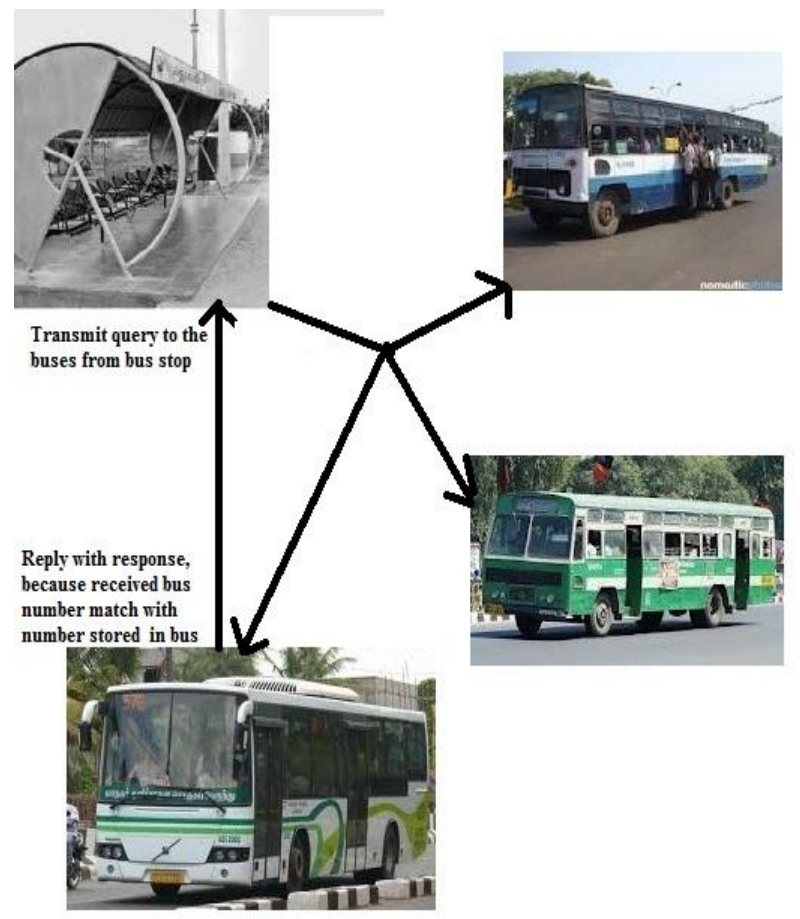

Figure 7: Over View of RTQS

The diagram represents the overview of entire Real Time Query System. Each bus stop has bus stop module and each bus has bus module. Bus stop module transmit query to all the buses. The bus whose number matches with the query number will send the response to the bus stop module.

\section{RESULT}

Figure 8 show the output obtained using the proposed approach. The display shows the stop number and the number of persons sitting inside the bus. At each stop, the bus stop number is displayed with the number of persons in the bus. The number of persons in the bus is updated at each bus stop.

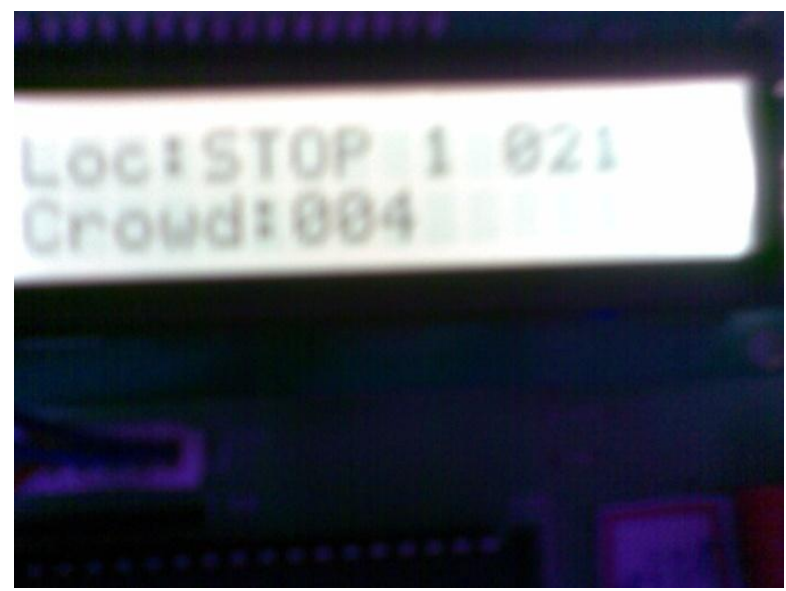

Figure 8: Output

\section{CONCLUSION}

The Real Time Query System is an efficient and low cost method in Intelligent Transport System (ITS).RTQS uses ZigBee for communication between bus module and bus stop module, the total cost of the system is greatly reduced. Over all power consumed by the system is also get reduced because of the ZigBee. This system is mainly used in urban areas where the traffic density is high. The system can fulfill the passengers demand in a better way. All the data are updated with latest information all the times which makes the Real Time Query System more information rich and reliable.

\section{REFERENCES}

[1] Vanajakshi, L., Subramanian, S.C. and Sivanandan, R., "Travel time prediction under heterogeneous traffic conditions using global positioning system data from buses", IET Intelligent Transport Systems, Volume: 3, Issue: 1, Page(s): 1 - 9, 2009.

[2] M.G.H. Bell, "Policy issues for the future intelligent road transport infrastructure", IEE Proceedings Intelligent Transport Systems, Volume:153, Issue: 2, Page(s): 147-155, 2006.

[3] Peter Tarasewich, Robert C. Nickerson and Merrill Warkentin, "Wireless/Mobile E-Commerce: Technologies, Applications, and Issues", Seventh Americas Conference on Information Systems, 2001.

[4] Roy, S.; Sen, R.; Kulkarni, S.; Kulkarni, P.; Raman, B.; Singh, L.K., "Wireless across road: RF based road traffic congestion detection", Third International Conference on Communication Systems and Networks (COMSNETS), Page(s): 1- 6, 2011.

[5] Seong Hoon Kim; Jeong Seok Kang; Hong Seong Park; Daeyoung Kim; Young-joo Kim, "UPnP-ZigBee internetworking architecture mirroring a multi-hop ZigBee network topology", IEEE Transactions on Consumer Electronics, Volume: $\quad$ 55, Issue: 3, Page(s): $1286 \quad$ - 1294 , 2009.

[6] Peng Ran; Mao-heng Sun; You-min Zou, "ZigBee Routing Selection Strategy Based on Data Services and Energy-Balanced ZigBee Routing", IEEE Asia-Pacific Conference on Services Computing (APSCC '06), Page(s): 400 - 404, 2006.

[7] Suzuki, N.; Mitani, T.; Shinohara, N., "Study and development of a microwave power receiving system for ZigBee device", Asia-Pacific Microwave Conference Proceedings (APMC), Page(s): 45- 48, 2010.'

[8] Jiung-yao Huang; Chung-Hsien Tsai, "Improve GPS positioning accuracy with context awareness", First IEEE International Conference on Ubi-Media Computing, Page(s): 94 - 99, 2008.

[9] Huaping Yu; Mei Guo, "A self-adapting data collection approach in wireless sensor networks for urban traffic information monitoring", IEEE International Conference on Intelligent Computing and Intelligent Systems (ICIS), Volume: 1, Page(s): 196 - 200, 2010. 
[10] Manolis.K., Kwstis.D, "Travelers Information Systems the case of a Medium Size City", Proc. IEEE Int. Conf. on Mechatronics, pp. 200204, 2004.

[11] Shalaby A., Farhan A., "Bus travel time prediction for dynamic operations control and passenger information systems", Proc. 82nd Ann. Meeting, Transportation, 2002.

[12] R.P.S. Padmanaban, K. Divakar, L. Vanajakshi, S.C. Subramanian,' "Development of a real-time bus arrival prediction system for Indian traffic conditions" Published in IET Intelligent Transport Systems, January 2010.

[13] Jeong R., Rilett L.R., "Bus arrival time prediction using artificial neural network model", IEEE Intelligent Transportation Systems Conf., pp. 988-993, 2004.

[14] Vaa, T.; Penttinen, M.; Spyropoulou, I., "Intelligent transport systems and effects on road traffic accidents: state of the art", IET Intelligent Transport Systems, Volume: 1, Issue: 2, Page(s): 81 - 88, 2007.

[15] Abe, K.; Tobana, T.; Sasamori, T.; Koizumi, H., "A study on a roadvehicle communication system for the future intelligent transport systems", Seventh International Conference on Parallel and Distributed Systems: Workshops, Page(s): 343 - 348, 2000. 